\title{
The Anterior Piriform Cortex Is Sufficient for Detecting Depletion of an Indispensable Amino Acid, Showing Independent Cortical Sensory Function
}

\author{
John B. Rudell, ${ }^{1}$ Adam J. Rechs, ${ }^{5}$ Todd J. Kelman, ${ }^{2}$ Catherine M. Ross-Inta, ${ }^{3}$ Shuzhen Hao, ${ }^{6}$ and Dorothy W. Gietzen ${ }^{4}$ \\ ${ }^{1}$ Physiology and Membrane Biology, School of Medicine, ${ }^{2}$ Preventive Veterinary Medicine Program, School of Veterinary Medicine, ${ }^{3}$ Department of \\ Medicine and Epidemiology, School of Veterinary Medicine, and ${ }^{4}$ Department of Anatomy, Physiology, and Cell Biology, School of Veterinary Medicine, \\ University of California, Davis, Davis, California 95616, 5 Department of Biological Sciences, Sacramento State University, Sacramento, California 95819, and \\ ${ }^{6}$ Ajinomoto, Inc., Fort Lee, New Jersey 07024
}

Protein synthesis requires a continuous supply of all of the indispensable (essential) amino acids (IAAs). If any IAA is deficient, animals must obtain the limiting amino acid by diet selection. Sensing of IAA deficiency requires an intact anterior piriform cortex (APC), but does it act alone? Shortly after rats begin eating an IAA-deficient diet, the meal ends and EPSPs are activated in the APC; from there, neurons project to feeding circuits; the meal ends within $20 \mathrm{~min}$. Within the APC in vivo, uncharged tRNA activates the general amino acid control non-derepressing 2 (GCN2) enzyme system increasing phosphorylation of eukaryotic initiation factor (P-eIF2 $\alpha$ ), which blocks general protein synthesis. If this paleocortex is sufficient for sensing IAA depletion, both neuronal activation and P-eIF $2 \alpha$ should occur in an isolated APC slice. We used standard techniques for electrophysiology and immunohistochemistry. After rats ate IAA-devoid or -imbalanced diets, their depleted slices responded to different stimuli with increased EPSP amplitudes. Slices from rats fed a control diet were bathed in artificial CSF replete with all amino acids with or without the IAA, threonine, or a tRNA synthetase blocker, L-threoninol, or its inactive isomer, D-threoninol. Thr depletion in vitro increased both EPSP amplitudes and P-eIF2 $\alpha$. L (but not D)-threoninol also increased EPSP amplitudes relative to control. Thus, we show independent excitation of the APC with responses parallel to those known in vivo. These data suggest a novel idea: in addition to classical processing of peripheral sensory input, direct primary sensing may occur in mammalian cortex.

\section{Introduction}

Maintenance of protein synthesis, which is crucial for survival in metazoans including mammals and birds, requires continuous availability of all the indispensable (essential) amino acids (IAAs). With IAA depletion, protein degradation begins rapidly (Kadowaki and Kanazawa, 2003). Rats detect a diet lacking any of the IAAs (Harper et al., 1970; Gietzen, 1993; Gietzen et al., 2007a) and reject it within the first meal (Koehnle et al., 2003). This adaptive response facilitates selection of a repleting diet (Gietzen and Rogers, 2006; Gietzen et al., 2007b). Both smell (Leung et al., 1972) and taste (Markison et al., 1999) have been ruled out as sensors of IAA depletion. Rather, the anterior piriform cortex (APC) is necessary for this anorectic response (Leung and Rogers, 1971; Beverly et al., 1990).

The question here is whether the APC acts alone in sensing IAA deficiency, without other neural input. A sensory function

\footnotetext{
Received Sept. 20, 2010; revised 0ct. 28, 2010; accepted Nov. 29, 2010.

This work was supported by National Institutes of Health Grants NS33347 and NS043210 and U.S. Department of Agriculture Grant 2000-01049. We are grateful for the advice and guidance of Dr. John M. Horowitz of University of California, Davis, throughout our electrophysiological studies.

D.W.G. has received support from Ajinomoto USA, Inc.

Correspondence should be addressed to Dr. Dorothy W. Gietzen, Department of Anatomy, Physiology, and Cell Biology, School of Veterinary Medicine, University of California, Davis, One Shields Avenue, Davis, CA 95616. E-mail: dwgietzen@ucdavis.edu.

DOI:10.1523/JNEUROSCI.4934-10.2011

Copyright $\odot 2011$ the authors $\quad 0270-6474 / 11 / 311583-08 \$ 15.00 / 0$
}

that originates in the cortex, is processed, and associated with a behavioral response has not, to our knowledge, been shown for an area of the cerebral cortex. Such a function could be shown if the responses of the isolated APC parallel those seen in the intact animal.

The biochemical responses to an IAA-deficient diet begin with a decreased concentration of the limiting IAA in the APC (Koehnle et al., 2004). The subsequent mechanisms for sensing IAA depletion include increased uncharged tRNA, which activates the general amino acid control non-derepressing (GCN) system (Hao et al., 2005). Amino acid alcohols compete for acylation of tRNA at the amino acid binding site (Cassio et al., 1967), increasing the levels of uncharged tRNA. Microinjection of L-threoninol, or L-leucinol, into the APC before presenting a control (basal) diet produces an anorectic response similar to that of an animal exposed to a diet lacking the respective IAA (Hao et al., 2005). This shows that either inhibition of, or limiting the substrate for, tRNA acylation can serve as the initial signal of an IAA deficiency; uncharged tRNA activates the GCN2 system (Anthony et al., 2001; Wek et al., 2006). Subsequent phosphorylation by GCN2 yields phosphorylated eukaryotic initiation factor $2 \alpha$ (P-eIF2 $\alpha$ ), which blocks protein synthesis at the level of initiation (Gietzen et al., 2004; Wek et al., 2006). Phosphorylation of eIF $2 \alpha$ serves as the biochemical readout for GCN2 activation. 
In vivo electrophysiology has shown increased EPSPs in the APC of animals fed an IAA-deficient diet (Hasan et al., 1998). Here, we recorded EPSPs in isolated APC slices, either ex vivo, from deficient animals or controls, or exposed to threonine (Thr)-deficient media in vitro. The slices were treated with various media to learn whether these same electrophysiological and biochemical responses would be seen absent other input, and whether they are associated with activation of the APC.

The results show totally independent sensory function for the APC in detecting depletion of the IAA, Thr. This demonstrates that a primary sensation, serving a homeostatic function of critical survival importance, may be included among the functions of the cerebral cortex.

\section{Materials and Methods}

Ex vivo studies: animals were fed a deficient diet before brain slice preparation

Animals. Male Sprague Dawley rats (Simonsen Laboratories) weighing $160-250 \mathrm{~g}$ were housed in individual hanging wire cages in a vivarium $\left(22^{\circ} \mathrm{C}\right)$ with a $12 \mathrm{~h} \mathrm{light/dark} \mathrm{cycle.} \mathrm{Animal} \mathrm{care} \mathrm{and} \mathrm{maintenance} \mathrm{fol-}$ lowed the National Institutes of Health guidelines, under a protocol approved by the Institutional Animal Care and Use Committee. The animals had ad libitum access to water and food. All animals ate a control diet for at least a week before receiving one of the experimental diets for $2 \mathrm{~h}$ to $20 \mathrm{~d}$, depending on the study.

Diets. The diets have been described in detail (Hammer et al., 1990; Koehnle et al., 2003). The basal diet (BAS) contained (w/w) 76\% carbohydrates (a 2:1 mixture of cornstarch/sucrose), 13\% amino acids (Ajinomoto), $5 \%$ corn oil (Mazola; Corn Products), 5\% minerals, $1 \%$ vitamins, and $0.1 \%$ choline chloride. The corrected (COR) diet included increased amounts of all IAAs. An isoleucine (Ile)-imbalanced (Ile-IMB) diet had increased amounts of all the IAAs, except for basal levels of Ile. The threonine-devoid (Thr-DEV), lysine-devoid (Lys-DEV), and histidinedevoid (His-DEV) diet formulations had increased amounts of the remaining IAAs but were devoid of the limiting IAA. In some studies, a $6 \%$ casein diet supplemented with methionine (so that the first limiting IAA became Thr) was used interchangeably with a Thr BAS diet. When amounts of the IAAs were altered, the carbohydrate mix was adjusted reciprocally, and the other ingredients, at levels designed to be fully replete, were unchanged.

For studies of IAA deficiency, any amino acid that is essential (indispensable in the diet) for mammals can be used as the growth-limiting amino acid, or "limiting IAA" (Harper et al., 1970). The amino acids that are essential for metazoans are remarkably similar (Gietzen and Rogers, 2006). In vivo feeding studies since the early 1900s have shown that we can "expect a marked failure in appetite when the diet is completely devoid of an essential [nitrogenous] component" (Rose, 1931). When rats eat a diet devoid of, or imbalanced in, an IAA, the concentrations of the limiting IAA in blood (Leung et al., 1968) and homogenates of the APC are reliably decreased (Gietzen et al., 1986, 1998; Koehnle et al., 2004). After the initial introduction of either a Thr-DEV or Leu-DEV diet, the reduced concentrations of the limiting IAA in the APC become significant by $21 \mathrm{~min}$ (Koehnle et al., 2004). The behavioral response to the lack of an IAA in the diet and the associated decrease in its concentration within the APC is a rapid termination of the first meal (e.g., within 20 min of exposure to a Thr-DEV diet) (Koehnle et al., 2003; Russell et al., 2003).

Various IAAs have been used in feeding experiments in animals (among the many reviews, see Harper et al., 1970; Rogers and Leung, 1973; Gietzen, 1993; Gietzen and Rogers, 2006). Virtually any IAA can be used as the limiting IAA for such studies, although mitochondrial metabolism can result in some storage of Lys (Benevenga and Blemings, 2007). In examples from our own work, we have used Thr and Ile (Gietzen et al., 1986), plus His (Gietzen et al., 1996), Lys (Hrupka et al., 1999), Leu (Hao et al., 2005) in the rat, and Trp in Limax (Gietzen et al., 1992).
Preliminary ex vivo studies used APC slices from rats fed IAA-depleting diets

For ex vivo studies, animals were given the dietary treatments in vivo, and then their APC slices were dissected and prepared for electrophysiological recording using the standard techniques detailed below. We had previously shown that norepinephrine (NE) is involved in the responses to Thr or Ile depletion in the APC (Gietzen et al., 1986). Therefore, in the first study (total $N=23$ ), controls were fed a stock pelleted diet (Purina; Nestle), and the experimental groups were given the His-DEV diet for $1 \mathrm{~d}$ $(n=3), 5 \mathrm{~d}(n=4), 7 \mathrm{~d}(n=3)$, or $20 \mathrm{~d}(n=5)$. Their APC slices were then tested for the amplitude of their response to NE as a measure of the excitability of the APC. In the second ex vivo trial, after several days on a basal diet, the experimental rats were given either an Ile-IMB diet $(n=4)$ or a COR diet $(n=3)$ for $2 \mathrm{~h}$; APC excitability was tested in these slices with input-output curves and the EPSP response to NE, and repletion of Ile in vivo and ex vivo.

In the third ex vivo study, BAS, COR, Thr-DEV, and Lys-DEV diets were used, and the slice treatments were amino acids in the media bathing the slices. The six groups (total $N=37$ ) were assigned according to DIET/slice treatment as follows: BAS/Thr $(n=7), \mathrm{COR} / \mathrm{Thr}(n=6)$, Thr-DEV/Thr $(n=6)$, Thr-DEV/Ser $(n=8)$, Lys-DEV/Lys $(n=4)$, and Lys-DEV/Thr $(n=6)$.

Brain slice preparation. The rats were decapitated, and their brains were quickly removed and placed in chilled artificial CSF ( $\mathrm{aCSF}$ ) for $\sim 30$ s. After careful removal of the dura mater, a transverse cut was made approximately at the level of the optic chiasm. Using the cut surface of the anterior portion of the brain as a base, a horizontal cut was made at the level of the olfactory bulb. The remaining ventral section of the forebrain, containing the APC, was then glued to an agar block with cyanoacrylate (Loctite). The tissue was bathed in chilled aCSF that had been equilibrated with carboxygen $\left(95 \% \mathrm{O}_{2}, 5 \% \mathrm{CO}_{2}\right)$. Slices $(400 \mu \mathrm{m})$ were cut from the submerged brain segment using a microtome (Vibroslice; World Precision Instruments). The slices were made $18^{\circ}$ from a true transverse section, perpendicular to the pial surface, to preserve the integrity of the apical dendrites of the pyramidal cells in layer II and the deep pyramidal cells (Jung et al., 1990). Four to five slices, rostral to the closure of the anterior commissure, $\sim 3.9$ to $\sim 1.9 \mathrm{~mm}$ rostral to bregma (Sharp et al., 2006), were retained. Slices were placed into aCSF at room temperature $\left(24-26^{\circ} \mathrm{C}\right)$, with continuous carboxygen bubbling, to recover for at least $2 \mathrm{~h}$ before recording.

The aCSF solution contained the following (in mM): $124.0 \mathrm{NaCl}, 3.5$ $\mathrm{KCl}, 2.0 \mathrm{CaCl}_{2}, 2.0 \mathrm{MgSO}_{4}, 26.0 \mathrm{NaHCO}_{3}, 1.3 \mathrm{NaH}_{2} \mathrm{PO}_{4}$, and 11.0 D-glucose. All additives were dissolved in aCSF and prepared $\sim 30 \mathrm{~min}$ before their bath application. Threonine (Thr), isoleucine (Ile), and serine (Ser) were from Ajinomoto; lysine (Lys) free-base and NE were from Sigma-Aldrich. The standard recording chamber (Fine Science Tools) had a well volume of $2 \mathrm{ml}$. Nylon mesh above and below the slice held it in place, submerged in a carboxygenated aCSF solution maintained at $30.5^{\circ} \mathrm{C}$ and perfused at a rate of $1 \mathrm{ml} / \mathrm{min}$.

Electrophysiology. Bipolar tungsten stimulating electrodes were visually placed $\sim 0.2 \mathrm{~mm}$ apart in layer III of the APC, deep to the lateral olfactory tract (LOT) and layer II. A glass microelectrode (1-3 M $\Omega$ ) filled with $3 \mathrm{M} \mathrm{NaCl}$ was then placed in layer III $\sim 0.5 \mathrm{~mm}$ lateral to the recording electrodes. With illumination from below, distinct translucence of the pyramidal cell bodies in layer II aided visual placement of the electrodes.

Slices were stimulated using $100 \mu$ s pulses (Acupulser stimulator; World Precision Instruments) at a constant current necessary to elicit a half-maximal field potential response. Levels of stimulation ranged from 35 to $65 \mu \mathrm{A}$. Waveforms were acquired by an Intra767 electrometer (World Precision Instruments) and amplified by a Grass preamplifier. Computer software customized with the ASYST programming package (Asyst Software) was used for recording and measuring the data. One waveform was collected every $3 \mathrm{~min}$ for the duration of the experiment, averaging the responses to 16 pulses delivered at $10 \mathrm{~s}$ intervals.

After establishing a stable baseline, the slice was perfused with a $10 \mathrm{~mm}$ concentration of either Thr, Lys, Ile, or Ser for 30 min or $30 \mu \mathrm{M}$ NE for 20 min. After washout of the added substances, restoration of baseline was 


\begin{tabular}{|c|c|c|c|}
\hline & aCSFThr-DEV (mM) & aCSFcomp (mм) & aCSFL- or D-throl (mM) \\
\hline $\mathrm{NaCl}$ & 112.936 & 112.936 & 112.936 \\
\hline $\mathrm{KCl}$ & 3.487 & 3.487 & 3.487 \\
\hline $\mathrm{NaH}_{2} \mathrm{PO}_{4} \cdot \mathrm{H}_{2} \mathrm{O}$ & 1.254 & 1.254 & 1.254 \\
\hline $\mathrm{MgSO}_{4}$ & 2.002 & 2.002 & 2.002 \\
\hline $\mathrm{NaHCO}_{3}$ & 25.949 & 25.949 & 25.949 \\
\hline Dextrose & 8.326 & 8.326 & 8.326 \\
\hline $\mathrm{CaCl}_{2}$ & 2.0 & 2.0 & 2.0 \\
\hline Alanine & 0.022 & 0.022 & 0.022 \\
\hline Arginine & 0.402 & 0.402 & 0.402 \\
\hline Asparagine $\cdot \mathrm{H}_{2} \mathrm{O}$ & 0.005 & 0.005 & 0.005 \\
\hline Cysteine & 0.01 & 0.01 & 0.01 \\
\hline Glutamine & 0.503 & 0.503 & 0.503 \\
\hline Glycine & 0.4 & 0.4 & 0.4 \\
\hline Histidine $\mathrm{HCl} \cdot \mathrm{H}_{2} \mathrm{O}$ & 0.2 & 0.2 & 0.2 \\
\hline Isoleucine & 0.8 & 0.8 & 0.8 \\
\hline Leucine & 0.8 & 0.8 & 0.8 \\
\hline Lysine $\mathrm{HCl}$ & 0.898 & 0.898 & 0.898 \\
\hline Methionine & 0.201 & 0.201 & 0.201 \\
\hline Phenylalanine & 0.4 & 0.4 & 0.4 \\
\hline Proline & 0.067 & 0.067 & 0.067 \\
\hline Serine & 0.4 & 0.4 & 0.4 \\
\hline Tryptophan & 0.078 & 0.078 & 0.078 \\
\hline Tyrosine & 0.397 & 0.397 & 0.397 \\
\hline Valine & 0.802 & 0.802 & 0.802 \\
\hline Threonine & - & 0.798 & 0.798 \\
\hline L,D-ThrOH & - & - & 8.179 \\
\hline Total & 162.339 & 163.137 & 171.316 \\
\hline
\end{tabular}

The few drops of $\mathrm{HCl}$ and or $\mathrm{NaCl}$ used in correcting the $\mathrm{pH}$ varied and so are not reflected in these values. Abbreviations: aCSFThr-DEV, Thr-devoid medium; aCSFcomp, complete amino acid medium; aCSFL-throl or aCSFDthrol, complete medium containing L- or D-threoninol, respectively.

obtained in each trial. Slices not exhibiting a stable baseline were omitted from the data set.

Statistics. Baseline was established by averaging the eight data points (48 min) immediately preceding each treatment and was used to normalize the data. Experimental values are presented as percentage changes from baseline, mean \pm SEM. Using the Statistical Analysis System (SAS), version 6.04, we performed ANOVA with post hoc evaluation of differences among treatment groups by least significant difference (LSD) tests after a significant $(p<0.05)$ ANOVA according to Snedecor and Cochran (1967). Where just two groups were compared, Student's $t$ test was used.

In vitro studies: rats were fed a control diet; slices were depleted of Thr in vitro

Animals. Similar rats $(N=25)$ were paired and housed in plastic cages in the same vivarium conditions as above. Fresh water and either Thr BAS or a $6 \%$ casein diet, described above, were available ad libitum immediately after arrival and for at least $5 \mathrm{~d}$ before use. Brains were taken and slices prepared as described above, except that the control aCSF included a complete set of both indispensable (IAA) and dispensable amino acids (aCSFcomp) (Table 1), adapted from Brewer et al. (1993), as used previously (Blais et al., 2003). In this study, Thr was the limiting IAA and L-threoninol (with D-threoninol as the inactive stereoisomer) was the blocker of threonyl-tRNA charging.

Electrophysiology. After recovery, one hemislice was placed in the recording chamber as above, bathed in aCSFcomp $\left(2 \mathrm{ml}, 29^{\circ} \mathrm{C}\right)$. The stimulating electrode was lowered into the LOT. A glass recording electrode (1.4-2.4 M $\Omega$ ) was then lowered into layer III of the APC, medial to the LOT. In this study, the levels of stimulation were $0.3-0.6 \mathrm{~mA}$. The averaged evoked potentials were recorded with LabVIEW (National Instruments). After a stable baseline response was established with the slice bathed in aCSFcomp (60-90 min), the medium was changed to one of the following: aCSFcomp (control) $(n=4)$, aCSFcomp without Thr
(aCSFThr-DEV) $(n=11)$, aCSFcomp plus D-threoninol (aCSFDthrol) $(n=4)$, or aCSFcomp plus L-threoninol (aCSFLthrol) $(n=6)$. Averaged evoked potentials continued to be recorded for 60-90 $\mathrm{min}$, when the medium was returned to aCSFcomp and responses were recorded for at least $180 \mathrm{~min}$ to allow a return to baseline.

In vitro media. Media (Table 1) were prepared from a stock solution (1-4l) consisting of aCSFcomp minus Thr. Amino acids were from Ajinomoto, Sigma-Aldrich or Fluka. The stock solution was then adjusted to $\mathrm{pH} \sim 7.4 \pm .01$ using an Accumet $\mathrm{pH}$ meter (Fisher Scientific) by adding $\mathrm{HCl}$. To obtain aCSFcomp, Thr (Fluka) was added to a portion of the stock solution. Likewise, to obtain aCSFL-throl or aCSFD-throl, L- or D-threoninol (Sigma-Aldrich) was added to aCSFcomp. After the addition of L- or D-threoninol, the $\mathrm{pH}$ of the medium was again adjusted to $\sim 7.4 \pm 0.01$. To account for the change in osmolarity as a result of adding $\mathrm{HCl}$, the same molar equivalents of $\mathrm{NaCl}$ were added to the aCSFcomp used in the L- or D-threoninol experiments.

Statistics for in vitro electrophysiology. Responses are presented as the percentage change from the baseline averaged evoked potential amplitude (in millivolts) \pm SEM. Baseline amplitudes were calculated as the average of the 10 average evoked potential amplitudes immediately before introducing the test medium. Each response was normalized to its own baseline amplitude. Normalized responses were calculated as the average of 10 consecutive average evoked potential amplitudes, beginning $12 \mathrm{~min}$ after the medium was switched (sufficient time for the new medium in the perfusion chamber to become thoroughly mixed), divided by the baseline amplitude and reported as a percentage change from baseline. The results for EPSP amplitude attributable to addition of the aCSFThr-DEV medium were compared with those attributable to addition of a complete medium (aCSFcomp) using one-way ANOVA. We compared the results for L-threoninol versus D-threoninol in the same manner. Responses were considered significant if $p<0.01$. Additionally, the effect of adding L-threoninol to the bath was determined by Cohen's $d$ test, in which the difference of the mean percentage change in EPSP between two experimental groups was divided by the pooled SD of the two groups. Values for a Cohen's " $d$ " effect size $>0.8$ are considered large, those between 0.8 and 0.5 are considered moderate, and those $<0.5$ are considered small effects (Cohen, 1994).

In vitro slice preparation for Western blotting. Slices were prepared as described for in vitro electrophysiology above. Each slice provided two identical APCs for comparison, as the structures are bilaterally symmetrical. The full slices were bisected along the midline; one side of each slice was used for the control and the other side for the experimental treatment. After recovery, the 24 hemislices taken from 12 APCs (three full slices from each of four rats) were incubated in either aCSFcomp or aCSFThr-DEV. Media included the phosphatase inhibitor mixtures 1 (p2850) and 2 (p5726) (Sigma-Aldrich). Mixture 1 inhibits the L-isozymes of alkaline phosphatases and Ser/Thr protein phosphatases (PPs) such as PP1 and PP2A. Mixture 2 inhibits acid and alkaline phosphatase and tyrosine protein phosphatases. After incubation for $5 \mathrm{~min}$ based on preliminary time course trials and as recommended by Hou and Klann (2004), the tissue was prepared for immunoblotting as described previously (Sharp et al., 2002).

We tested phosphoprotein indicators of IAA sensing, as determined in previous whole-animal studies (Gietzen et al., 2004; Sharp et al., 2004). To demonstrate activity of the GCN2 kinase, we used the phosphorylated (P) forms of its substrate, eIF2 $\alpha$ (Gietzen et al., 2004), and to indicate activation of the neurons (Sharp et al., 2004), we used phosphorylated calcium/calmodulin kinase II (P-CaMKII). Western blotting, as described previously (Gietzen et al., 2004), used the following antibodies: phosphorylated P-eIF2 $\alpha$ [rabbit polyclonal IgG (P-eIF2 $\alpha$; phosphorylated on S51); Cell Signaling Technology; diluted 1:1000]; P-CaMKII [rabbit polyclonal IgG (anti-active CaMKII pT286); Promega; diluted 1:2000]. To determine protein loading in each lane, after scanning for the phosphoprotein of interest, blots were stained with Coomassie blue dye and rescanned. Phosphoprotein density absorbancies (relative to the protein level determined for each band and expressed as relative density units) provide the outcome data. 


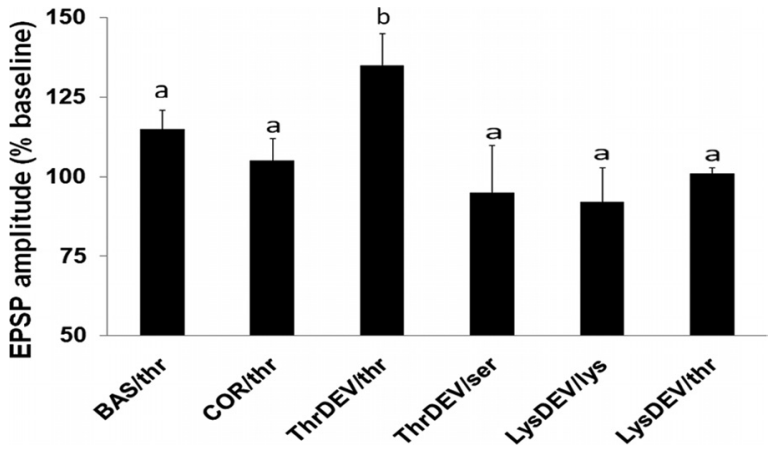

Figure 1. Effects of amino acids on field potential amplitude in slices from rats prefed in vivo. Threonine added to the slice medium increased EPSP amplitudes only in APC slices from rats previously fed a Thr-DEV diet. For each amino acid trial, the eight data points (48 min) immediately preceding drug application were averaged and used as a baseline value. Groups, by in vivo diet/ex vivo slice treatment (BAS/Thr, COR/Thr, Thr-DEV/Thr, Thr-DEV/Ser, Lys-DEV/Lys, and Lys-DEV/Thr), are indicated on the abscissa. Each trial provided its own baseline control. The bars represent treatment means, and the vertical lines represent SEM. Treatments having different letters differ significantly $(p<0.05)$.

Statistics for the Western blots. Each value was normalized to the protein measurement for its own gel. To test for differences between the devoid-treated and control APC hemislices, we used a two-way ANOVA with replications using slice location and IAA treatment as factors, with the Data Analysis Toolkit, Microsoft Office Excel 2003, followed by the LSD test for differences among the treatments, as above. The $\alpha$ level for significance was set at $p \leq 0.05$.

\section{Results}

Ex vivo results: IAA depletion was accomplished in vivo Both His-DEV and Ile-IMB diets potentiated the APC ex vivo In the first study, APC slices from the His-DEV diet groups showed greater EPSP responses to NE than the controls (control, $28.9 \pm 1.75 \%)$. The increases after His-DEV were as follows: day $1,+7.44 \%$; day $5,+10.9 \%$; day $7,+4.7 \%$; and day $20,+10.2 \%$. The increases seen for days 1, 5, and 20 of His-DEV feeding were significant at $p<0.05$. In the smaller Ile-IMB trial, after $2 \mathrm{~h}$ on the IMB diet, the best slice showed that, even after only $2 \mathrm{~h}$, a $10 \%$ increase in the NE response could be seen. Slices from rats that had been repleted with the COR diet for $2 \mathrm{~h}$ returned to control levels of the response to NE (IMB, 34.6\%; vs COR, 24.6\%). The EPSP amplitudes were significantly $(26 \pm 0.01 \% ; p<0.05)$ higher for the IMB group $(n=4)$ versus the COR group $(n=3)$ in the responses to inputs from 300 to $700 \mathrm{~mA}$. When Ile was repleted in the medium, even higher output was seen; the average increase in EPSP amplitude was $27 \pm 5 \%$.

Activation of Thr-deficient tissue slices by repletion of Thr in vitro In the third ex vivo study, when Thr was applied to the slices from the Thr-DEV diet group, there was a significant (overall $F_{(5,31)}=$ 2.287; $p<0.05$ ) increase in field potential amplitude to $135 \pm$ $10 \%$ of baseline (Fig. 1). This result is in line with the Ile-IMB/Ile result noted above. This excitatory effect occurred $\sim 9$ min after bath application of Thr. Field potential amplitudes returned to baseline values $\sim 15 \mathrm{~min}$ after the Thr was washed out, showing full recovery after the IAA treatment. The addition of Thr to slices from the rats fed either BAS or COR had no effect on field potential amplitude: $115 \pm 6$ and $105 \pm 7 \%$ for BAS and COR, respectively. Addition of the dispensable amino acid Ser to Thr-DEV slices also had no effect on the field potential amplitudes $(95 \pm$ $15 \%$ of baseline), as expected. In contrast to the positive responses of the His-DEV, Ile-IMB, and Thr-DEV slices, there was no effect seen in slices taken from the Lys-DEV-fed rats (field

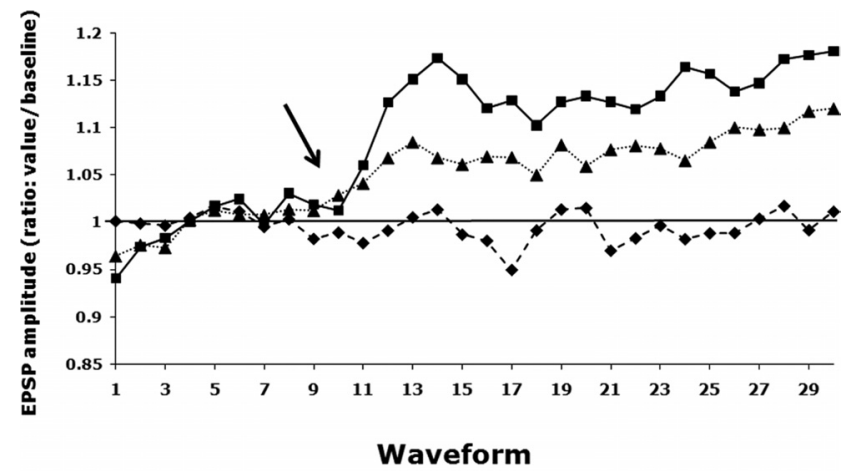

\section{- - Control $\rightarrow$-Devoid $\quad \cdots \cdots$ L-ThrOH}

Figure 2. EPSP amplitudes as percentage of baseline over time differ as a function of the in vitro slice medium. Each waveform (as indicated on the abscissa) represents 16 EPSPs averaged over $3 \mathrm{~min}$. The arrow is directed at the time the bathing medium was changed to aCSFThr-DEV (devoid), aCSFL-throl (L-ThrOH), or aCSFcomp (control), as indicated in the legend. The markers indicate means for EPSP amplitude expressed as a ratio of treatment amplitude/baseline, for that treatment at that interval.

potential amplitudes were $92 \pm 11$ and $101 \pm 2 \%$ of baseline for Lys and Thr, respectively).

\section{In vitro results: APC slices deprived of Thr in vitro}

APC slices are potentiated by Thr deprivation or L-threoninol in vitro

The isolated APC brain slice was potentiated by changes of the media that induced either Thr deficiency or deacylated tRNA. EPSP amplitudes were increased in layer III of the APC in response to the removal of an IAA (aCSFThr-DEV). The increase in EPSP amplitude attributable to aCSFThr-DEV began within 6 min of changing the medium and peaked after an average of 12 min (Fig. 2). EPSP amplitudes were increased to $112.9 \pm 2.1 \%$ of baseline with aCSFThr-DEV (Figs. 2, 3), compared with a change of $98.7 \pm 1.7 \%$ of baseline for the control, aCSFcomp $\left(F_{(1,13)}=\right.$ $14.07 ; p<0.01)$.

Addition of L-threoninol (aCSFL-throl) to the perfusion medium resulted in an increase of EPSP amplitude to $107.3 \pm 2.8 \%$ of baseline (Fig. 3), whereas the biologically inactive isomer of threoninol (D-threoninol; aCSFD-throl) had no effect (100.1 \pm $3.1 \%$ of baseline). Response to the addition of $\mathrm{L}$-threoninol began within $3 \mathrm{~min}$ and reached a plateau after $9 \mathrm{~min}$. There was a directional, albeit nonsignificant, effect with L-threoninol $(n=6)$ compared with D-threoninol $(n=4)\left(F_{(1,8)}=2.88 ; p=0.13\right)$. The response to D-threoninol also did not differ from the response to aCSFcomp $\left(F_{(1,6)}=0.13 ; p=0.73\right)$. Given the similarity between the two control treatments, aCSFcomp was used as the control for the response to L-threoninol. In this condition, the response to L-threoninol approached significance $\left(F_{(1,8)}=5.11\right.$; $p=0.054)$. Analysis by the more powerful Cohen's test (Cohen, 1994) revealed a large effect on EPSP amplitude $(d=1.1)$ attributable to L-threoninol. Importantly, the EPSP amplitudes for aCSFThr-DEV and L-threoninol did not differ $\left(F_{(1,15)}=2.47 ; p=0.14\right)$.

Location of responsive areas in the APC slices

Positive EPSP responses to Thr depletion were obtained in slices ranging from 3.9 to $1.9 \mathrm{~mm}$ rostral to bregma with the greatest and most frequent responses obtained from slices that were $\sim 2.7$ $\mathrm{mm}$ rostral to bregma (Fig. 4). This shows the anatomical specificity of the response, and localizes it to layers II and III of the 

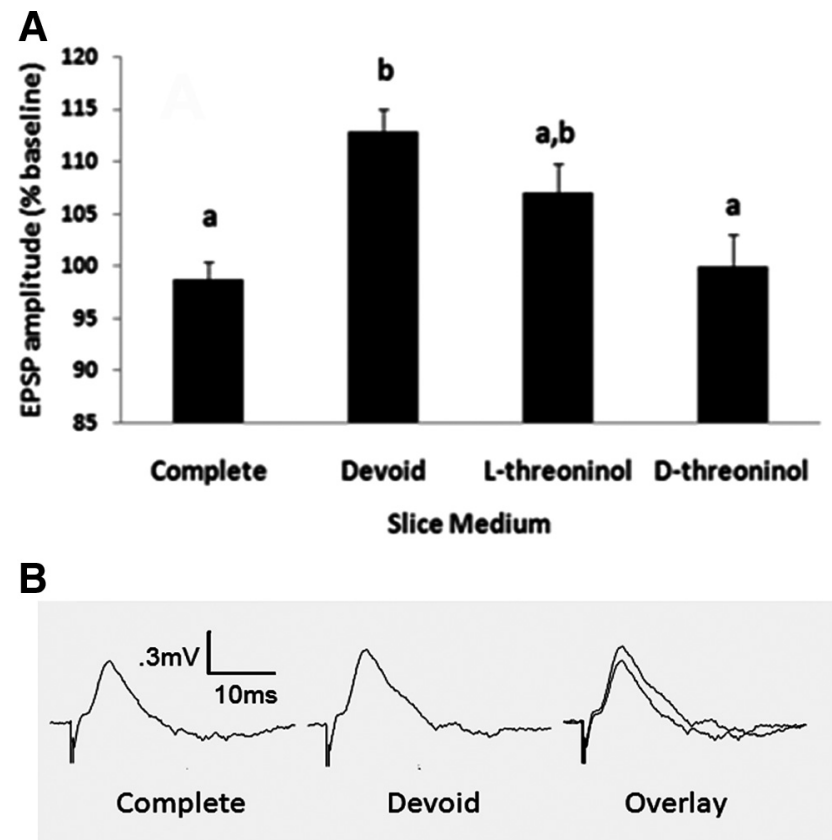

Figure 3. A, The average percentage change (from baseline) in EPSP amplitude was computed over a period of $30 \mathrm{~min}$, beginning $12 \mathrm{~min}$ after the slice bath was refreshed with each medium. EPSP amplitudes were increased in the Thr-devoid medium (devoid = aCSFThr-Dev) and in the presence of $\mathrm{L}$-threoninol, compared with the complete medium and D-threoninol, the inactive isomer. Slice media are indicated on the abscissa and are described in Table 1. Different lowercase letters indicate a significant difference $(p<0.01)$. The difference between L-threoninol and D-threoninol was analyzed using Cohen's test for effect size and found to be large $(d=1.1)$. $B$, Examples of the field potential (EPSP) recordings from the in vitro studies in the isolated APC for the control (complete $=\mathrm{aCSF}$ comp), threonine deficient (devoid $=$ a(SFThr-DEV), and overlay of both (right).

APC, consistent with our earlier immunochemical findings in tissue taken from rats fed a Thr-DEV diet (Sharp et al., 2006).

\section{Immunoblotting results}

In the in vitro brain slice both P-eIF $2 \alpha$ and P-CaMKII were increased by Thr depletion (Fig. 5). These slices depleted of Thr in vitro responded similarly to those in APC tissue taken after in vivo dietary Thr deficiency (Gietzen et al., 2004; Sharp et al., 2004). One animal's tissue was deleted from the data set because of outliers in the control samples for P-eIF $2 \alpha$; 9 slices yielding 18 APC hemislices remained in the P-eIF2 $\alpha$ data set. There was no interaction between slice location and IAA treatment, or statistical difference attributable to the slice location (for location: $F_{(2,12)}=1.96, p=0.18$ ). The volume densities (normalized to band protein) for P-eIF $2 \alpha$ were significantly increased in the deficient slices $\left(F_{(1,12)}=6.04 ; p=0.03\right)$. The values for the slices taken together were as follows: control, $0.47 \pm 0.04$, and devoid, $0.67 \pm 0.07$. Taken separately by slice location, just the slices $2.4-1.6 \mathrm{~mm}$ rostral to bregma reached the level of the LSD, showing anatomical specificity for this phosphoprotein as well. The increases in P-CaMKII were greater; there was no interaction and no effect of slice location $\left(F_{(2,18)}=0.37 ; p=0.70\right)$. Normalized densities for P-CaMKII were as follows: control, $0.39 \pm 0.06$, and devoid, $0.93 \pm 0.11\left(F_{(1,18)}=16.85 ; p=0.0006\right)$; all three slice locations showed significant IAA effects according to the LSD.

\section{Discussion}

The APC acts alone in sensing depletion of Thr

Here, we show that the APC can function independently of other neural input in responding to depletion of the IAA, Thr. Both

\begin{tabular}{|c|c|c|}
\hline $\begin{array}{l}\text { Distance } \\
\text { from } \\
\text { Bregma }\end{array}$ & $\begin{array}{l}\text { Recording Electrode } \\
\text { Locations }\end{array}$ & $\begin{array}{l}\text { Percent Increase } \\
\text { from Baseline } \\
\text { Amplitude }\end{array}$ \\
\hline
\end{tabular}

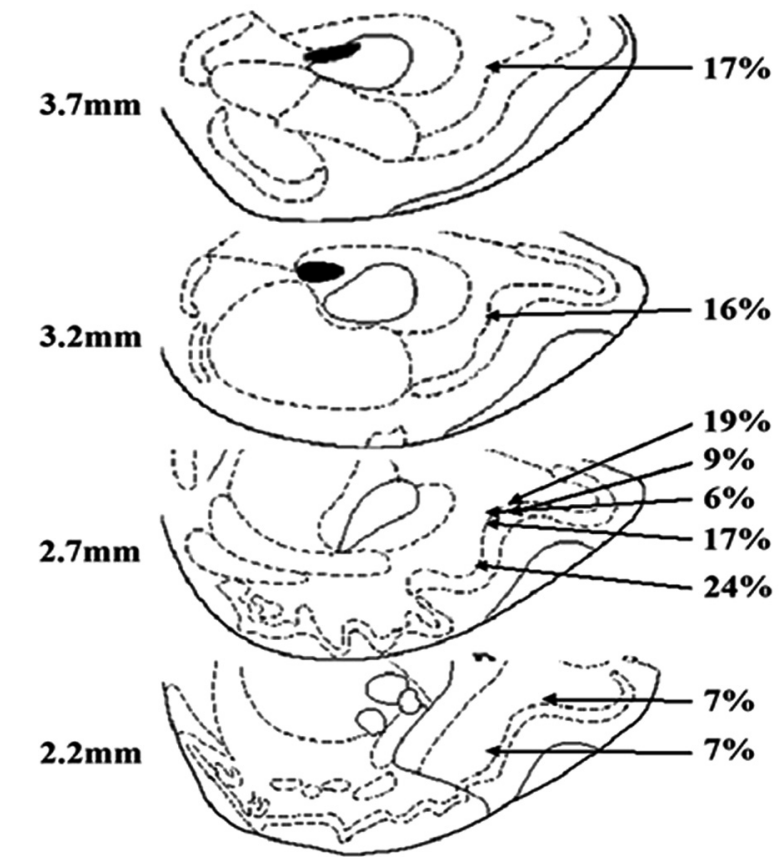

Figure 4. Locations of stimulating and recording electrodes, which yielded positive EPSP increases from baseline with the Thr-devoid medium (aCSFThr-DEV) in the APC slice studies, are similar to the most active sites found by Sharp et al. (2006). Approximate placements of the recording electrodes are shown with the corresponding percentage increase from baseline in EPSP amplitude in Thr deficiency (aCSFThr-DEV) as shown in Figures 2 and 3 . Slices were stimulated by an electrode placed in the lateral olfactory tract and EPSPs were recorded from layer III of the APC. Slice diagrams are arranged in order based on their distance rostral to bregma (Paxinos and Watson, 1986).

neuronal activation and the biochemical mechanisms that are involved in responding to IAA depletion, known from in vivo studies (Hasan et al., 1998; Gietzen et al., 2004; Hao et al., 2005), are seen in the isolated APC. These results demonstrate that the APC is not only necessary, as already known (Leung and Rogers, 1971; Rogers and Leung, 1973; Beverly et al., 1990), but sufficient for the sensory limb of the behavioral responses to depletion of the IAA, Thr.

APCs from animals depleted of IAA in vivo, and then treated as slices, can respond to IAAs

The first experiments reported here used animals given the IAAdepleting or control diets for various times before the study. The Thr-DEV group showed increased EPSP amplitudes with selective in vitro repletion of Thr, APC slices from the HisDEV group showed significantly greater responses to NE challenge, and the Ile-IMB slices were potentiated relative to COR and showed an increased EPSP response to added Ile. We saw the expected (Harper et al., 1970) control responses (i.e., no difference with BAS or COR diets, or to a nonlimiting IAA, or to Ser, a nonessential amino acid). That the Lys-Dev group did not respond to Lys in the medium could be attributable to the metabolism of Lys in mitochondria, making depletion more difficult to achieve (Hrupka et al., 1999; Benevenga and Blemings, 2007). It is important that the ex vivo studies demonstrated appropriate responses to deficiency of three different IAAs in an isolated slice, showing, as expected (Harper et al., 1970), that the in vitro responses are 

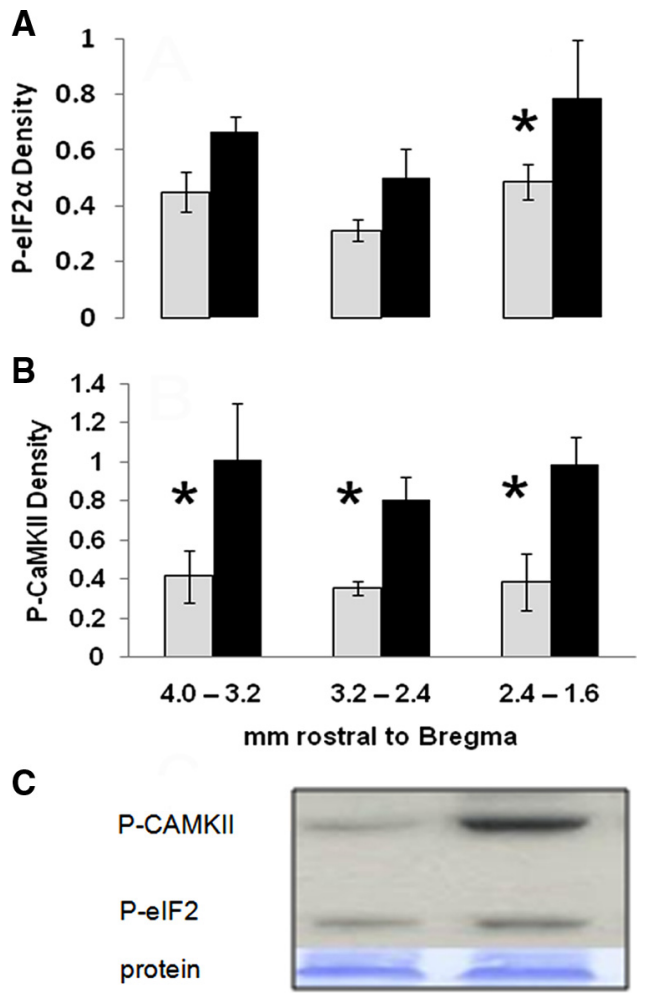

Figure 5. Phosphorylated proteins in APC tissue are increased after in vitro incubation with aCSFcomp (light bars) or aCSFThr-DEV (dark bars) for volume density units (density). Slice locations (in millimeters rostral to bregma) are indicated on the abscissa. $\boldsymbol{A}, \mathrm{P}$-elF2 $\alpha$. $\boldsymbol{B}$, P-CaMKII. C, Representative gel segment showing effects of aCSFcomp (left) or aCSFThr-DEV (right) on phosphoprotein densities, as indicted at the left of the gel segment. Values are bars, means, and horizontal lines, SE. The asterisks $\left(^{*}\right)$ indicate significant differences attributable to IAA treatment within the slice location $(p<0.05)$ by LSD after a significant overall ANOVA for this variable. At the bottom of the segment is the Coomassie blue protein, an indicator of equal loading in the gel.

not selective to any single IAA. This set the stage for the subsequent in vitro studies.

\section{In vitro responses to IAA depletion and repletion parallel those seen in vivo}

Whether the APC requires additional neural, neurochemical, or hormonal input for activation in IAA deficiency was not known. Mimicking dietary IAA depletion, although absent any difficulties that might be associated with the blood-brain barrier, by bathing an isolated APC slice with a medium devoid of an IAA should cause an increase neuronal activity. The increase in EPSP amplitude began within the first 6 min of Thr depletion and peaked after $\sim 12 \mathrm{~min}$, well before the time at which a behavioral response is observed. This timing further supports our hypothesis that activation of the APC is among the earliest events in the mechanisms underlying the behavioral response.

Similar anorectic behavior, to that seen with an IAA-DEV diet, was induced in intact rats by microinjecting the APC with L-threoninol and L-leucinol (Hao et al., 2005), which block the loading of an amino acid onto its cognate tRNA (Cassio et al., 1967). This increases the concentration of uncharged tRNA (Rouget and Chapeville, 1968). Because increasing uncharged threonyl-tRNA in vivo produces a behavioral response similar to that seen in rats fed either a Thr- or Leu-devoid diet, we expected that the in vitro electrophysiological response to an increase in uncharged threonyl-tRNA would be similar to that of the response to the removal of Thr. It was; addition of L-threoninol to the slice preparation did indeed increase EPSP amplitude.

The removal of Thr from aCSFcomp to prepare aCSFThrDEV and the addition of L-threoninol (for aCSFL-throl) differed slightly from aCSFcomp in their osmolarity because of the addition of solute to one and the removal of solute from the other. Since the treatments had the same effect on EPSP amplitude, we can conclude that these responses were not attributable to osmolarity. The important similarity between the two treatments is that they each cause an increase in the concentration of uncharged threonyl-tRNA.

\section{Biochemical responses to IAA depletion are seen in vivo and in vitro}

The activation of the "general amino acid control" (i.e., GCN2) system generalizes to depletion of any IAA (Kimball, 2002). Thus, the crucial findings from the present in vitro studies are that the isolated APC slice responds similarly to Thr depletion and to L (but not D)-threoninol, with both neuronal activation and increased phosphorylation of eIF $2 \alpha$. There are reports that the mammalian target of rapamycin (mTOR) responds to amino acids (Hara et al., 1998); however, we have shown that the mTOR system has no role in the responses of the APC to IAA deficiency (Hao et al., 2010). Rather, in vitro depletion of Thr results in increased P-eIF2 $\alpha$, indicating increased GCN2 activity in the APC, as does a Thr-DEV diet (Hao et al., 2005).

\section{Phosphoproteins in vitro replicate in vivo findings}

Because P-eIF $2 \alpha$ blocks the initiation of translation in general protein synthesis at the level of initiation (Anthony et al., 2001; Wek et al., 2006), this increase in excitability is consistent with the loss of rapidly turning-over inhibitory elements that cannot be replaced without new protein synthesis. Consistent with this, the $\beta 3$ subunit of the GABA receptor is downregulated $2 \mathrm{~h}$ after an IAA-imbalanced meal (Truong et al., 2002). Also, the area of the APC that appears to house the sensor for IAA deficiency, as shown in Figure 4, here, and in the study by Sharp et al. (2006), overlaps with the "area tempestas," which has limited inhibitory capabilities, making it highly chemosensitive (Ekstrand et al., 2001). An additional depletion of inhibitory elements could easily exacerbate the activation of the APC circuitry.

\section{Brain circuits involved in the responses to IAA depletion}

The APC receives synaptic input both from the olfactory bulb and central sources (Shepherd, 1979; Shipley et al., 2008). As noted above, olfaction is not involved in the recognition of IAA depletion (Leung et al., 1972). However, central inputs arrive as well (Shepherd, 1979; Gietzen, 1993; Gietzen et al., 2007a). Knife cuts around the dorsal medial nucleus of the hypothalamus (DMN) increased intake of IAA-imbalanced diets (Bellinger et al., 1999), suggesting that the DMN might have a role in sensing IAA deficiency. Fibers bearing numerous neurotransmitters are found in the APC (Haberly and Bower, 1989; Ennis et al., 2007). These were shown to affect the feeding responses to IAA-deficient diets using a variety of neurotransmitter antagonists, microinjected into the APC, to block the anorectic response (Gietzen and Magrum, 2001). Yet the present findings show that these inputs are not necessary for the sensory limb of this response. Finally, although cortical neurons can be sensitive to calcium (Yano et al., 2004), protons (Mari et al., 2010), or hypoxia (Hochachka et al., 1996), 
the sensory receptor for a behavioral homeostatic response has not been located to cortex, as far as we know.

In its role as an association cortex (Haberly and Price, 1978; Shepherd, 1979), the APC allows integration of information about IAA depletion with other food-related signals. Moreover, as well as housing the IAA deficiency-sensing mechanism, the APC is uniquely situated to integrate olfaction and taste (Shepherd, 2006).

The APC projects to many brain areas, including the hypothalamus, which is part of the feeding circuitry (Berthoud, 2002). On activation of the APC, glutamatergic signaling from the output cells in layer III of the APC (Shipley et al., 2008) projects to feeding-related areas, including the lateral hypothalamus ( $\mathrm{LH})$ (Blevins et al., 2004) in a suggested neural circuit (Gietzen et al., 1998), indicating to the animal that it is acquiring insufficient amounts of IAA and that a new source must be obtained. In line with this, the $\mathrm{LH}$ is activated $30 \mathrm{~min}$ after injection of small amounts of Thr into the APC of a Thr-deficient animal (Monda et al., 1997); these results, along with the DMH lesion studies discussed above (Bellinger et al., 1999), provide clear evidence of the involvement of APC-hypothalamic projections in the subsequent responses to IAAs.

\section{Cortical sensing occurs absent peripheral sensors: a novel role for the cortex?}

This study establishes an in vitro excitatory response, in cortex, to an increase in uncharged tRNA after a decrease in a limiting IAA (Thr in the in vitro studies here), or inhibition of tRNA charging with the stereospecific form of threoninol. Increased P-eIF $2 \alpha$ in the slices after in vitro incubation with a Thr-devoid medium further shows the role of the "general amino acid control," GCN2, pathway in this model, and the even greater increase in $\mathrm{P}-\mathrm{CaMKII}$ indicates that secondary activation spreads within the APC circuitry. Therefore, the APC initiates the neuronal activity associated with IAA depletion and that signaling of IAA depletion must originate in the APC.

We conclude that the APC can be activated in isolation by depletion of an IAA, in this case, Thr, absent any other sensory or neural input. The traditional concept of sensory function holds that a stimulus activates its receptor in the periphery; only then is the transduced message projected to higher centers, including the cortex, for processing. In striking contrast, this work shows that the APC, which has been known to be necessary for some 40 years, is also sufficient for detecting depletion of Thr. These findings lead us to offer a novel concept: a direct intrinsic sensory function for the mammalian cortex.

\section{References}

Anthony TG, Reiter AK, Anthony JC, Kimball SR, Jefferson LS (2001) Deficiency of dietary EAA preferentially inhibits mRNA translation of ribosomal proteins in liver of meal-fed rats. Am J Physiol Endocrinol Metab 281:E430-E439.

Bellinger LL, Evans JF, Tillberg CM, Gietzen DW (1999) Effects of dorsomedial hypothalamic nuclei lesions on intake of an imbalanced amino acid diet. Am J Physiol 277:R250-R262.

Benevenga NJ, Blemings KP (2007) Unique aspects of lysine nutrition and metabolism. J Nutr 137:1610S-1615S.

Berthoud HR (2002) Multiple neural systems controlling food intake and body weight. Neurosci Biobehav Rev 26:393-428.

Beverly JL, Gietzen DW, Rogers QR (1990) Effect of dietary limiting amino acid in prepyriform cortex on food intake. Am J Physiol 259:R709-R715.

Blais A, Huneau JF, Magrum LJ, Koehnle TJ, Sharp JW, Tomé D, Gietzen DW (2003) Threonine deprivation rapidly activates the system A amino acid transporter in primary cultures of rat neurons from the essential amino acid sensor in the anterior piriform cortex. J Nutr 133:2156-2164.
Blevins JE, Truong BG, Gietzen DW (2004) NMDA receptor function within the anterior piriform cortex and lateral hypothalamus in rats on the control of intake of amino acid-deficient diets. Brain Res 1019:124-133.

Brewer GJ, Torricelli JR, Evege EK, Price PJ (1993) Optimized survival of hippocampal neurons in B27-supplemented Neurobasal, a new serumfree medium combination. J Neurosci Res 35:567-576.

Cassio D, Lemoine F, Waller JP, Sandrin E, Boissonnas RA (1967) Selective inhibition of aminoacyl ribonucleic acid synthetases by aminoalkyl adenylates. Biochemistry 6:827-836.

Cohen J (1994) The earth is round ( $p<0.05)$. Am Psychol 49:997-1003.

Ekstrand JJ, Domroese ME, Johnson DM, Feig SL, Knodel SM, Behan M, Haberly LB (2001) A new subdivision of anterior piriform cortex and associated deep nucleus with novel features of interest for olfaction and epilepsy. J Comp Neurol 434:289-307.

Ennis M, Hamilton KA, Hayar A (2007) Neurochemistry of the main olfactory system. In: Handbook of neurochemistry and molecular biology: sensory neurochemistry (Lajtha A, Johnson DA, eds), pp 139-204. New York: Springer.

Gietzen DW (1993) Neural mechanisms in the responses to amino acid deficiency. J Nutr 123:610-625.

Gietzen DW, Magrum LJ (2001) Molecular mechanisms in the brain involved in the anorexia of branched-chain amino acid deficiency. J Nutr 131:851S-855S.

Gietzen DW, Rogers QR (2006) Nutritional homeostasis and indispensable amino acid sensing: a new solution to an old puzzle. Trends Neurosci 29:91-99.

Gietzen DW, Leung PM, Rogers QR (1986) Norepinephrine and amino acids in prepyriform cortex of rats fed imbalanced amino acid diets. Physiol Behav 36:1071-1080.

Gietzen DW, Harris AS, Carlson S, Gelperin A (1992) Amino acids and serotonin in Limax maximus after a tryptophan devoid diet. Comp Biochem Physiol A Comp Physiol 101:143-149.

Gietzen DW, Dixon KD, Truong BG, Jones AC, Barrett JA, Washburn DS (1996) Indispensable amino acid deficiency and increased seizure susceptibility in rats. Am J Physiol 271:R18-R24.

Gietzen DW, Erecius LF, Rogers QR (1998) Neurochemical changes after imbalanced diets suggest a brain circuit mediating anorectic responses to amino acid deficiency in rats. J Nutr 128:771-781.

Gietzen DW, Ross CM, Hao S, Sharp JW (2004) Phosphorylation of eIF2alpha is involved in the signaling of indispensable amino acid deficiency in the anterior piriform cortex of the brain in rats. J Nutr 134:717-723.

Gietzen DW, Hao S, Anthony TG (2007a) Amino acid sensing mechanisms: biochemistry and behavior. In: Handbook of neurochemistry and molecular neurobiology: sensory neurochemistry (Lajtha A, Johnson DA, eds), pp 249-269. New York: Springer.

Gietzen DW, Hao S, Anthony TG (2007b) Mechanisms of food intake repression in indispensable amino acid deficiency. Annu Rev Nutr 27:63-78.

Haberly LB, Bower JM (1989) Olfactory cortex: model circuit for study of associative memory? Trends Neurosci 12:258-264.

Haberly LB, Price JL (1978) Association and commissural fiber systems of the olfactory cortex of the rat. J Comp Neurol 178:711-740.

Hammer VA, Gietzen DW, Sworts VD, Beverly JL, Rogers QR (1990) Adrenal hormones and the anorectic response and adaptation of rats to amino acid imbalance. J Nutr 120:1617-1623.

Hao S, Sharp JW, Ross-Inta CM, McDaniel BJ, Anthony TG, Wek RC, Cavener DR, McGrath BC, Rudell JB, Koehnle TJ, Gietzen DW (2005) Uncharged tRNA and sensing of amino acid deficiency in mammalian piriform cortex. Science 307:1776-1778.

Hao S, Ross-Inta CM, Gietzen DW (2010) The sensing of essential amino acid deficiency in the anterior piriform cortex, that requires the uncharged tRNA/GCN2 pathway, is sensitive to wortmannin but not rapamycin. Pharmacol Biochem Behav 94:333-340.

Hara K, Yonezawa K, Weng QP, Kozlowski MT, Belham C, Avruch J (1998) Amino acid sufficiency and mTOR regulate p70 S6 kinase and eIF-4E BP1 through a common effector mechanism. J Biol Chem 273:14484-14494.

Harper AE, Benevenga NJ, Wohlhueter RM (1970) Effects of ingestion of disproportionate amounts of amino acids. Physiol Rev 50:428 -558.

Hasan ZA, Woolley DE, Gietzen DW (1998) Responses to indispensable 
amino acid deficiency and replenishment recorded in the anterior piriform cortex of the behaving rat. Nutr Neurosci 1:373-381.

Hochachka PW, Buck LT, Doll CJ, Land SC (1996) Unifying theory of hypoxia tolerance: molecular/metabolic defense and rescue mechanisms for surviving oxygen lack. Proc Natl Acad Sci U S A 93:9493-9498.

Hou L, Klann E (2004) Activation of the phosphoinositide 3-kinase-Aktmammalian target of rapamycin signaling pathway is required for metabotropic glutamate receptor-dependent long-term depression. J Neurosci 24:6352-6361.

Hrupka BJ, Lin Y, Gietzen DW, Rogers QR (1999) Lysine deficiency alters diet selection without depressing food intake in rats. J Nutr 129:424-430.

Jung MW, Larson J, Lynch G (1990) Long-term potentiation of monosynaptic EPSPs in rat piriform cortex in vitro. Synapse 6:279-283.

Kadowaki M, Kanazawa T (2003) Amino acids as regulators of proteolysis. J Nutr 133:2052S-2056S.

Kimball SR (2002) Regulation of global and specific mRNA translation by amino acids. J Nutr 132:883-886.

Koehnle TJ, Russell MC, Gietzen DW (2003) Rats rapidly reject diets deficient in essential amino acids. J Nutr 133:2331-2335.

Koehnle TJ, Russell MC, Morin AS, Erecius LF, Gietzen DW (2004) Diets deficient in indispensable amino acids rapidly decrease the concentration of the limiting amino acid in the anterior piriform cortex of rats. J Nutr 134:2365-2371.

Leung PM, Rogers QR (1971) Importance of prepyriform cortex in foodintake response of rats to amino acids. Am J Physiol 221:929-935.

Leung PM, Rogers QR, Harper AE (1968) Effect of amino acid imbalance on plasma and tissue free amino acids in the rat. J Nutr 96:303-318.

Leung PM, Larson DM, Rogers QR (1972) Food intake and preference of olfactory bulbectomized rats fed amino acid imbalanced or deficient diets. Physiol Behav 9:553-557.

Mari Y, Katnik C, Cuevas J (2010) ASICla channels are activated by endogenous protons during ischemia and contribute to synergistic potentiation of intracellular $\mathrm{Ca}^{2+}$ overload during ischemia and acidosis. Cell Calcium 48:70-82.

Markison S, Gietzen DW, Spector AC (1999) Essential amino acid deficiency enhances long-term intake but not short-term licking of the required nutrient. J Nutr 129:1604-1612.

Monda M, Sullo A, De Luca V, Pellicano MP, Viggiano A (1997) L-threonine injection into PPC modifies food intake, lateral hypothalamic activity, and sympathetic discharge. Am J Physiol 273:R554-R559.
Paxinos G, Watson C (1986) The rat brain in stereotaxic coordinates, Ed 2. Sydney: Academic.

Rogers QR, Leung PM (1973) The influence of amino acids on the neuroregulation of food intake. Fed Proc 32:1709-1719.

Rose WC (1931) Feeding experiments with mixtures of highly purified amino acids. 1. The inadequacy of diets containing nineteen amino acids. J Biol Chem 94:155-165.

Rouget P, Chapeville F (1968) Reactions sequence of leucine activation catalysed by leucyl-RNA synthetase. 1. Kinetic studies. Eur J Biochem 4:305-309.

Russell MC, Koehnle TJ, Barrett JA, Blevins JE, Gietzen DW (2003) The rapid anorectic response to a threonine imbalanced diet is decreased by injection of threonine into the anterior piriform cortex of rats. Nutr Neurosci 6:247-251.

Sharp JW, Magrum LJ, Gietzen DW (2002) Role of MAP kinase in signaling indispensable amino acid deficiency in the brain. Brain Res Mol Brain Res 105:11-18.

Sharp JW, Ross CM, Koehnle TJ, Gietzen DW (2004) Phosphorylation of $\mathrm{Ca}^{2+} /$ calmodulin-dependent protein kinase type II and the alphaamino-3-hydroxy-5-methyl-4-isoxazole propionate (AMPA) receptor in response to a threonine-devoid diet. Neuroscience 126:1053-1062.

Sharp JW, Ross-Inta CM, Hao S, Rudell JB, Gietzen DW (2006) Colocalization of phosphorylated extracellular signal-regulated protein kinases 1/2 (ERK1/2) and phosphorylated eukaryotic initiation factor 2alpha (eIF2alpha) in response to a threonine-devoid diet. J Comp Neurol 494:485-494.

Shepherd GM (1979) Olfactory cortex. In: The synaptic organization of the brain, Ed 2 (Shepherd GM, ed), pp 289-307. New York: Oxford UP.

Shepherd GM (2006) Smell images and the flavour system in the human brain. Nature 444:316-321.

Shipley MT, Ennis M, Puche AC (2008) The olfactory system. In: Neuroscience in medicine (Conn M, ed), pp 611-622. Totowa, NJ: Humana.

Snedecor GW, Cochran WG (1967) Statistical methods, Ed 6. Ames, IA: Iowa State UP.

Truong BG, Magrum LJ, Gietzen DW (2002) GABA $_{A}$ and GABA $A_{B}$ receptors in the anterior piriform cortex modulate feeding in rats. Brain Res 924:1-9.

Wek RC, Jiang HY, Anthony TG (2006) Coping with stress: eIF2 kinases and translational control. Biochem Soc Trans 34:7-11.

Yano S, Brown EM, Chattopadhyay N (2004) Calcium-sensing receptor in the brain. Cell Calcium 35:257-264. 